\title{
Estimation of Chest Compression Rate and Detection of Hands-off Intervals During Resuscitation With Automated External Defibrillators
}

\author{
Jesus Ruiz ${ }^{1}$, Sofía Ruiz de Gauna ${ }^{1}$, Pablo Bahillo ${ }^{1}$, Digna M González-Otero², Purificación Saiz ${ }^{1}$, \\ Karlos Ibarguren ${ }^{3}$, Daniel Alonso ${ }^{3}$ \\ ${ }^{1}$ University of the Basque Country, Bilbao, Spain \\ ${ }^{2}$ Osatu S Coop, Bexen Cardio, Ermua, Spain \\ ${ }^{3}$ The Basque Country Emergency Medical Services System, San Sebastián, Spain
}

\begin{abstract}
Quality of chest compressions (CCs) is key for patient's survival to cardiac arrest. Incorporating real-time feedback on CC rate in automated external defibrillators (AEDs) could enhance CC quality. The aim of the study was to design a method for detecting CC pauses and computing CC rate using the transthoracic impedance (TI) signal acquired from the AED defibrillation pads.

We collected 35 AED recordings from a database property of Emergentziak-Osakidetza (Basque Country, Spain). Using the ECG and TI signals, a CC pause was annotated when CCs were interrupted for more than $3 \mathrm{~s}$. The algorithm estimates the CC rate from the autocorrelation of 2-s consecutive TI intervals.

The algorithm reported a sensitivity (Se) and a positive predictive value $(\mathrm{PPV})$ in the detection of CC pauses of 97.64\%/92.48\%. Reliable CC rates were obtained with a Se/PPV of $99.03 \% / 98.61 \%$. Unreliable CC rates were reported only in $0.9 \%$ of the cases.

A method based on the autocorrelation of the TI signal allows reliable detection of CC pauses and estimation of $C C$ rates, enhancing current $A E D$ functionality.
\end{abstract}

\section{Introduction}

Early high-quality cardiopulmonary resuscitation (CPR) and early access to defibrillation are key to maximize survival from out-of-hospital cardiac arrest [1]. CPR consists on delivering chest compressions (CCs) and ventilations to the patient in order to generate a minimal but critical amount of blood flood to the vital organs. Defibrillation is the application of an electric current through the heart to disrupt its disorganized electrical activity in order to restore a perfusing rhythm.

In the last decades, public access defibrillation programs have been deployed to make automated external defibrillators (AEDs) available to lay people [2]. AEDs are portable and simple to operate devices that analyze the victim's electrocardiogram (ECG) to determine whether a shockable rhythm is present. AEDs can also reliably guide the resuscitation sequence through voice and/or visual prompts.

However, high-quality CPR is difficult to achieve in the resuscitation field even by well-trained rescuers [3]. Current resuscitation guidelines indicate that CCs must be delivered to the patient with minimal pauses (also named hands-off intervals), at a rate of 100-120 compressions per minute, and with a depth of 50-60 mm [1]. Adherence to these goals is difficult; hands-off intervals are very frequent and there is a tendency to provide too fast chest compressions, diminishing depth accordingly $[3,4]$.

Besides the ECG, most AEDs record the transthoracic impedance (TI) signal through the defibrillation pads. Chest compression activity can be observed in the TI signal, since CCs cause fluctuations around the patient's baseline impedance with an amplitude about one ohm [5]. The aim of the study was to design a method for detecting CC pauses and computing CC rate using the TI signal acquired from the AED defibrillation pads.

\section{Materials and methods}

\subsection{Database description}

We selected thirty-five (35) AED recordings (one per patient) from out-of-hospital cardiac arrest events with more than 1000 CCs applied to each patient. Recordings were collected between 2013 and 2014 by the basic life support (BLS) of Emergentziak-Osakidetza, the emergency medical services system of the Basque Country (Spain). Data were provided anonymous.

Recordings were acquired with LIFEPAK1000 AEDs (Physio-Control, USA). ECG and TI signals were acquired with sampling frequencies of $125 \mathrm{~Hz}$ and $60 \mathrm{~Hz}$, respectively. The raw ECG was band-pass filtered to suppress direct current and high frequency noise prior to its storage in the defibrillator's memory. Similarly, the raw TI signal was high-pass filtered to suppress patient's 
baseline impedance. Concurrent ECG and TI waveforms of each recording were resampled to $250 \mathrm{~Hz}$ and exported to Matlab (Mathworks, USA) format.

\subsection{Data annotation}

When providing CPR, the BLS applied 2 min series of compressions following the $30: 2$ pattern, that is, alternating 30 CCs followed by a pause for delivering 2 ventilations [1]. After every 2 min, the AED prompts the user to interrupt CCs for rhythm analysis. With this sequence in mind, we reviewed the ECG and the TI signals of each recording, and manually annotated a pause if CCs were interrupted for more than $3 \mathrm{~s}$.

Figure 1 shows a 1 min interval of an AED recording. The ECG depicted at the top indicates that the patient presents ventricular fibrillation (a shockable rhythm). The TI signal depicted below shows fast oscillations of amplitudes in the range 0.5 to $1 \mathrm{ohm}$. Those fluctuations disappear when CCs are discontinued, so CC pauses can be easily distinguished in the TI signal.
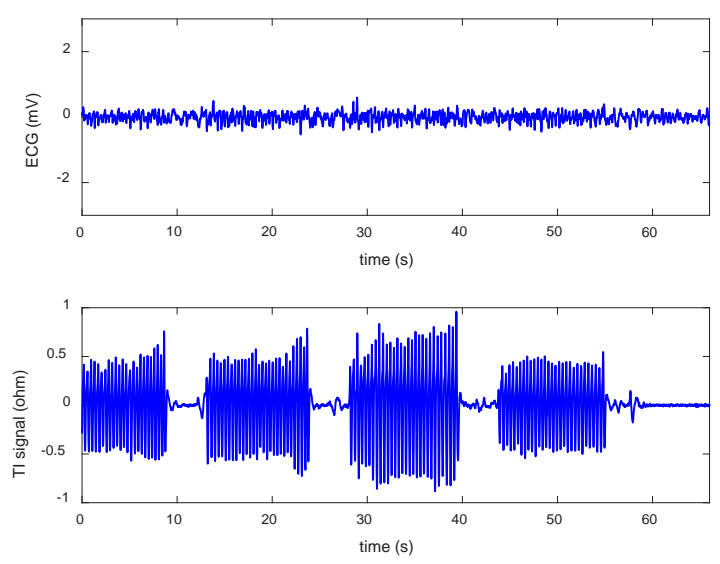

Figure 1. Example of the two signals, ECG (top) and TI, acquired by most AEDs. The patient presents a shockable rhythm, and the TI signal allows the identification of the CPR sequence, consisting of series of 30 compressions with pauses for ventilation in between.

\subsection{Description of the method}

The algorithm processes the TI signal through consecutive non-overlapped $2 \mathrm{~s}$ analysis windows. The algorithm computes a biased estimate of the autocorrelation of each analysis window, and analyzes the lag range from 0 to $0.8 \mathrm{~s}$. The method locates a peak in the autocorrelation signal between $0.25 \mathrm{~s}$ and $0.8 \mathrm{~s}$, of amplitude higher than a defined threshold. The interval of interest in the autocorrelation implies that the algorithm was designed to detect CC rates in the range between 75 compressions per minute (cpm) and $240 \mathrm{cpm}$, according to the expression:

$$
f_{c c}(\mathrm{cpm})=60\left(\frac{\mathrm{s}}{\min }\right) \cdot \frac{1}{T(\mathrm{~s})}
$$

If there is no peak satisfying the amplitude condition in the established range, the value of CC rate assigned for the analysis window is $f_{c c}=0$, and the interval is determined as "interval with no CCs". Otherwise, a non-zero $f_{c c}$ value corresponded to the "interval with CCs", i.e. it is not a CC pause.
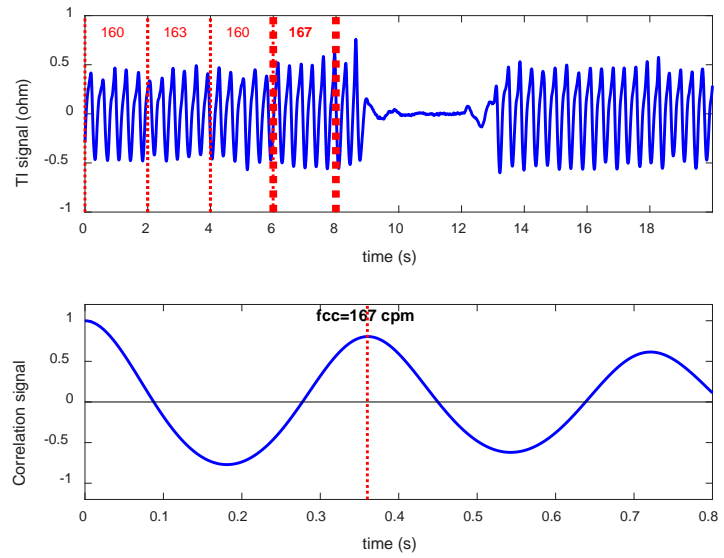

Figure 2. Example of the algorithm's operation in the presence of CCs. For each 2 s analysis window, the computed CC rate is depicted (top). For the analysis window corresponding to the 6-8 s interval, the TI autocorrelation (bottom) shows the detected peak which corresponds to a CC rate of $167 \mathrm{cpm}$.
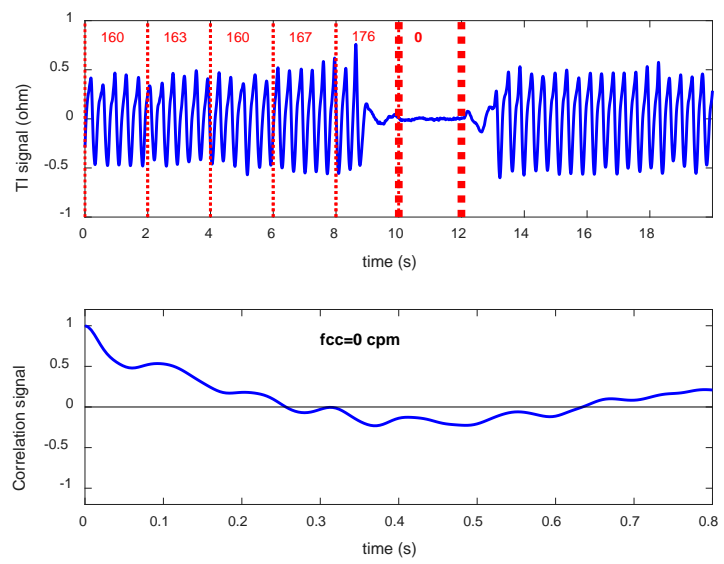

Figure 3. Detection of a CC pause. For the analysis window corresponding to the 10-12 s interval, no significant peak was detected in the TI autocorrelation, so the algorithm accurately reported $f_{c c}=0$. 


\subsection{Performance evaluation}

Recordings were split into a training (10 recordings) and a test set. First, the method was evaluated in terms of its ability to detect CC pauses, defined as intervals longer than $3 \mathrm{~s}$ in the absence of CCs. For that aim, sensitivity (Se) and positive predictive value (PPV) were selected as figures of merit. Se was defined as the percentage of annotated pauses detected by the algorithm; PPV was defined as the percentage of detected pauses that corresponded to a true pause.

Second, the method was evaluated in terms of its ability to reliably estimate CC rate. In the available data, there was no gold standard for validating the CC rate values reported by the algorithm. That limitation impeded the computation of the error in the measurement of CC rates. Alternatively, we used Se and PPV as figures of merit to evaluate the correctness in the estimation of CC rate for each analysis window. Thus, Se indicated the percentage of annotated CCs intervals for which a non-zero CC rate was reported, and PPV the percentage of reported non-zero CC rate values truly corresponding to compression intervals.

In addition, we manually reviewed all computed CC rate values in the compression series for the test set, and unreliable values far from the visually measured CC rates were annotated.

\section{Results}

A total of 1611 CC pauses were annotated in the test set, with a median (IQR) of 63 (45-81) CC pauses per episode. With the test set, the method reported a Se/PPV in the detection of CC pauses of $97.64 \% / 92.48 \%$. The computation of CC rates was achieved with a Se/PPV of 99.03\%/98.61\%. Unreliable CC rates were reported only in $0.9 \%$ of the cases, and were typically associated with an underestimation of the $\mathrm{CC}$ rate, usually corresponding to half of the observed rate.

The following figures present some examples of interest that illustrate the algorithm's performance. Generally, the algorithm performed very well but sometimes it did not work as expected. Figure 4 shows an example of an annotated CC pause that was not detected. In that case, small fluctuations can be observed in the TI signal, and the algorithm reported a non-zero $f_{c c}$ value.

Figure 5 shows an example of a single $f_{c c}=0$ reported by the algorithm in a series of CCs (analysis window corresponding to the interval 938-940 s). Nevertheless, the algorithm correctly detects the presence of CCs in the previous and following 2-s windows of the compression series.
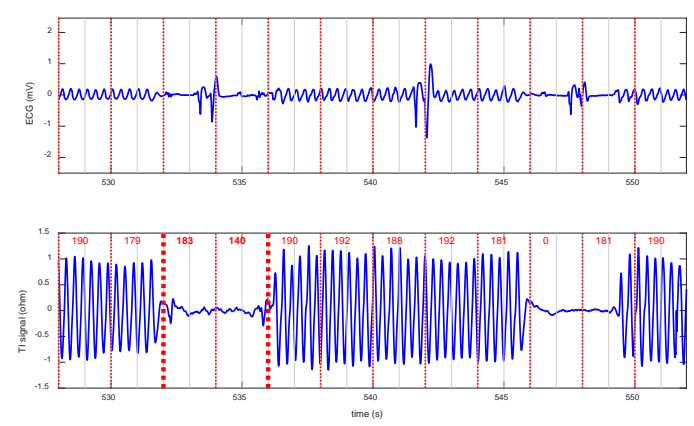

Figure 4. Example of a non-detected pause: $f_{c c} \neq 0$ in two consecutive analysis windows of an interval annotated as a pause (532-536 s).
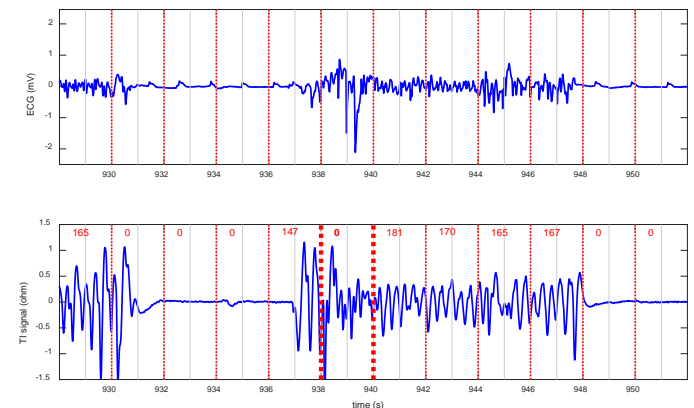

Figure 5. The algorithm reports a single $f_{c c}=0$ in a series of CCs (938-940s).

Finally, Figure 6 shows an example of the underestimation of the CC rate. In that particular case, the failure occurred in a $2 \mathrm{~s}$ interval almost at the end of the compression series, and a reliable CC rate was reported in the following window.
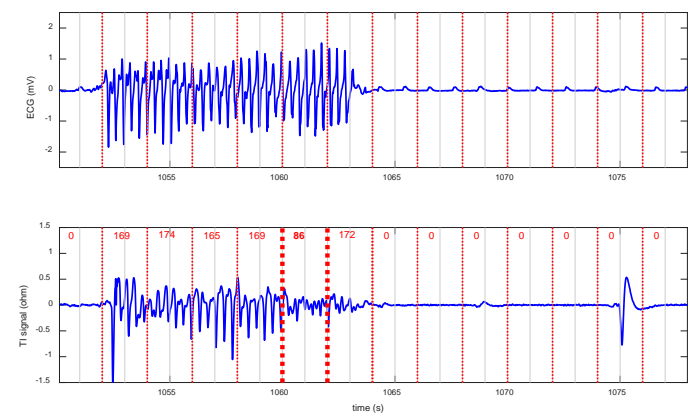

Figure 6. Example of an unreliable CC rate value (1060$1062 \mathrm{~s}$ ). CC rate is underestimated (approximately half the real value is reported). 


\section{Discussion}

Most current AEDs do not incorporate real-time help on the quality of CPR. Enhancing AEDs to guide rescuers during chest compressions would be a significant step forward to achieve high-quality CPR by the BLS, including lay people as first responders.

Hands-off intervals are frequent during out-of-hospital $\mathrm{CPR}$. Interruptions in chest compressions compromise blood flow to the heart and brain and decrease defibrillation success, and, consequently, survival. Our method reliably detects pauses in CCs. The automated detection of such pauses could be relevant for two main reasons. First, in the field of CPR quality, it would enable AEDs to provide feedback to the rescuer when too long interruptions in CCs are detected. Second, it would allow detecting intervals in which the ECG is not affected by the artifact induced by CCs (e.g. pauses for ventilation or rescuer switch). In those intervals, AEDs could reliably assess the ECG rhythm, without requiring an additional interruption of compressions for rhythm assessment.

The algorithm showed a good performance in the estimation of CC rate. Feedback on CC rate would help rescuers to adhere to the $100-120 \mathrm{cpm}$ range currently recommended. In addition, AED storage of continuous CC rate would be very useful for episode debriefing. However, we need to test the algorithm with data incorporating a reference signal (e.g. the compression depth signal obtained from a CPR feedback device) to be used as gold standard for assessing the accuracy of the method in the computation of CC rate.

Finally, the algorithm could be implemented in current AEDs with minimal software modifications, without requiring additional devices.

\section{Conclusions}

High-quality CPR is key in the effective treatment of out-of-hospital cardiac arrest. Most current AEDs cannot guide rescuers to provide chest compressions in adherence to recommendations.

A method based on the autocorrelation of the TI signal, currently acquired through defibrillation pads, could allow the reliable detection of compression pauses and estimation of compression rates, thus enhancing AED functionality.

\section{Acknowledgments}

This work was supported by the Basque Government (Basque Country, Spain), through the grant IT1087-16. The authors thank Emergentziak-Osakidetza BLS providers for collecting the data used in this study.

\section{References}

[1] K. G. Monsieurs, J. P. Nolan, L. L. Bossaert et al., "European Resuscitation Council guidelines for resuscitation 2015. Section 1. Executive summary," Resuscitation, vol. 95, pp. 1-80, Oct. 2005.

[2] M. L. Weisfeldt, R. E. Kerber, R. P. McGoldrick et al., "Public access defibrillation. A statement for healthcare professionals from the American Heart Association Task Force on Automatic External Defibrillation," Circulation, vol. 92, no. 9, pp. 2763, Nov. 1995.

[3] P. A. Meaney, B. J. Bobrow, M. E. Mancini et al., "Cardiopulmonary resuscitation quality: improving cardiac resuscitation outcomes both inside and outside the hospital: a consensus statement from the American Heart Association," Circulation, vol. 128, no. 4, pp. 417-435, Jul. 2013.

[4] L. Wik, J. Kramer-Johansen, H. Myklebust et al., "Quality of cardiopulmonary resuscitation during out-of-hospital cardiac arrest,” JAMA, vol. 293, no. 3, pp. 299-304, Jan. 2005.

[5] F.S. Stecher, J.A. Olsen, R.E. Stickney, and L. Wik, "Transthoracic impedance used to evaluate performance of cardiopulmonary resuscitation during out of hospital cardiac arrest," Resuscitation, vol. 79, no. 3, pp. 432-437, Dec. 2008.

Address for correspondence.

Sofía Ruiz de Gauna

University of the Basque Country, UPV/EHU

School of Engineering, Building 1

Plaza Torres Quevedo, 1 48013-Bilbao (Spain)

sofia.ruizdegauna@ehu.eus 